\title{
Simple Eye Closure Penalty Estimate for Amplitude Noise-Degraded Signals
}

\author{
Terence Broderick and Sonia Boscolo \\ Photonics Research Group, School of Engineering and Applied Science, \\ Aston University, B4 7ET, United Kingdom. \\ broderit@aston.ac.uk
}

\begin{abstract}
We present a simplified model for a simple estimation of the eye-closure penalty for amplitude noise-degraded signals. Using a typical 40-Gbit/s return-to-zero amplitude-shift-keying transmission, we demonstrate agreement between the model predictions and the results obtained from the conventional numerical estimation method over several thousand kilometers.
\end{abstract}

Keywords: Eye Diagram, On-Off Keying, Gaussian Statistics, Eye Closure Penalty

\section{INTRODUCTION}

The increase of the capacity of communication systems can be achieved by an increase in the channel bit-rate. Increasing the channel rate assumes the utilization of shorter carrier pulses. It is well known that the propagation of short pulses is strongly affected by the fibre dispersion, which results in large temporal broadening of the carrier pulses. Subsequently, the carrier pulse power spreads over many time slots and so the accumulated effect of the instantaneous fibre nonlinearity tends to get averaged out. Signal transmission using short pulses is known as the pseudo-linear regime

Fibre nonlinearity within pseudo-linear transmission of RZ-OOK pulses is dominated by Intra-channel Four-Wave Mixing (IFWM) [1], leading to eye closure due to amplitude fluctuations of logical ones and ghost pulses generated within logical zeros [1]. The evaluation of the penalty due to such nonlinearity is of great interest due to such systems being in demand in accordance with growing requirements for capacity and increased bit-rate.

The numerical modelling of such complex lightwave communication systems is an effective and well-used tool for design and performance evaluation. While accurate, this type of simulation can also be lengthy and computationally expensive for modern fibre-optic communication links, which are comprise a number of components using a variety of modulation formats and operational regimes. For this reason, it is sometimes desirable to obtain estimates of the effects of impairments that cause signal degradation by the relatively simple evaluation of the eye closure (EC) in the signal eye diagram [2-5].

The effects of impairments that can be assessed by propagation of optical channels without noise such as fibre dispersion or without any actual propagation distance at all such as optical filtering have been studied with simulation and analysis in the recent past by evaluation of the eye-closure penalty (ECP) [2-5]. In most cases, the results have been related to the Q-factor penalty or the power penalty [2-4].

Analytical or semi-analytical results for the system performance evaluation are of great importance even if they are derived based on simplified models. In this paper, we present a simple theoretical method for the estimation of the expected ECP for signals whose quality is mainly deteriorated by the amplitude noise accumulated during transmission due to IFWM [1]

Optical Transmission, Switching, and Subsystems VI, edited by Ken-ichi Kitayama, Pierpaolo C. Ghiggino, Kim Roberts,

Yikai Su, Proc. of SPIE Vol. 7136, 71362D · @ 2008 SPIE · CCC code: 0277-786X/08/\$18 · doi: 10.1117/12.803527 


\section{THEORETICAL MODEL}

RZ-OOK data formatted pulses are vulnerable to two types of nonlinearity, IFWM and intra-channel Cross-Phase Modulation (IXPM) [1]. Dominant amongst both phenomena is IFWM, which manifests as amplitude fluctuations in logical ones and noise in logical zeros. Such distortion allows the assumption of symmetric signal degradation on the eye diagram. Statistically, RZ-OOK transmission is not governed by Gaussian models [7, 8], but the BER can be estimated to good accuracy using Gaussian statistics due to symmetry of the eye closure [9].

We begin by noting the definition for EC. Supposing the centre of the bit slot is the location of maximum pulse amplitude, an appropriate measure of EC is provided by the difference between the innermost rails of the signal electrical eye diagram $[2,3]$ :

$$
E C=\min \left[V_{1}\right]-\max \left[V_{0}\right]
$$

where $\min \left[V_{1}\right]$ is the voltage level of the minimum "ones" rail at the eye centre, and $\max \left[V_{0}\right]$ is the voltage level of the maximum "zeros" rail. Let us assume now a Gaussian shape for the received-voltage distributions of the "ones" and "zeros", an approximation that usually works relatively well in communication systems using the conventional ASK modulation format [8]. We can then use simple rules governing the extrema of Gaussian distributed samples to derive an upper bound for the EC. We know that:

$$
\begin{aligned}
& \min \left[V_{1}\right] \leq E\left[V_{1}\right]-3 \sigma_{V_{1}} \\
& \max \left[V_{0}\right] \geq E\left[V_{0}\right]+3 \sigma_{V_{0}}
\end{aligned}
$$

Where $E\left[V_{1}\right], \sigma_{1}$, and $E\left[V_{0}\right], \sigma_{0}$ are the average voltage and the standard deviation of the "ones" and "zeros" distributions, respectively. Thus, it is straightforward to write

$$
E C \leq E\left[V_{1}\right]-E\left[V_{0}\right]-3\left(\sigma_{V_{1}}+\sigma_{V_{0}}\right) .
$$

The ECP can then be calculated as the ratio of the EC before transmission to this quantity after transmission [2]:

$$
E C P=10 \log _{10}\left(E C_{\text {in }} / E C_{\text {out }}\right) \text {. }
$$

An example of a degraded signal eye is illustrated schematically in Fig. 1.

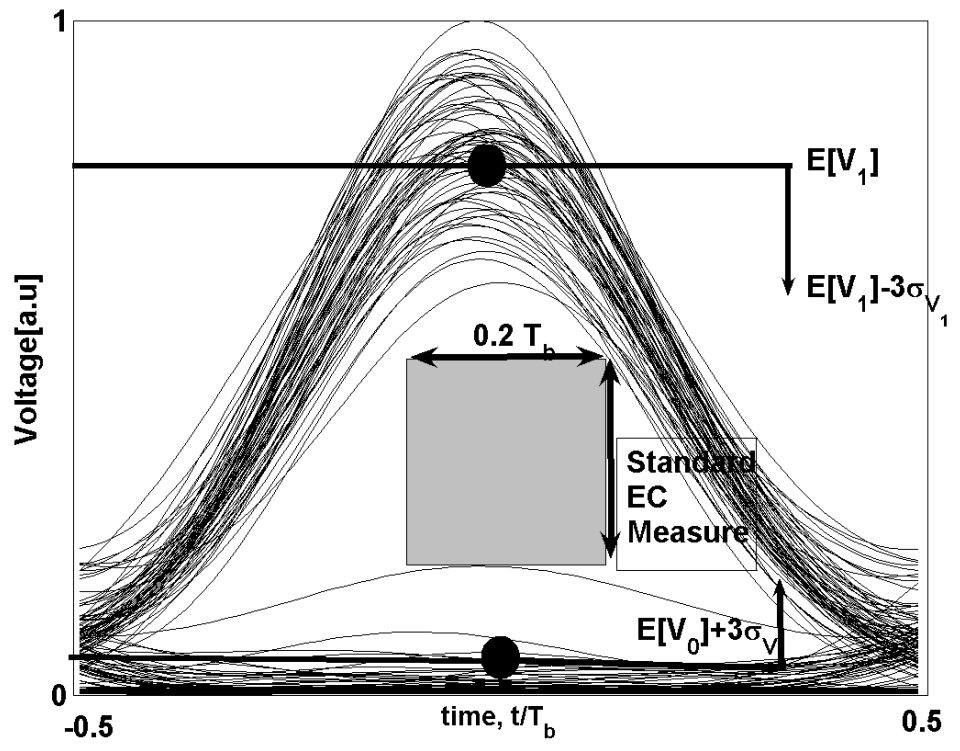

Figure 1: Example of a degraded signal electrical eye diagram and eye-closure estimate. 
Note that the validity of the derived EC estimate for general signal degradation primarily rests on the assumption that the signal eye is closed symmetrically from the top and bottom. Thus, the effects of timing jitter and of waveform distortions such as those originated from strong inter-symbol interference (ISI) and inter-channel crosstalk of wavelength-division multiplexed systems cannot be accounted for by the underlying model. The model has potential applicability in ASK systems when the dominant source of signal degradation is amplitude noise.

\section{NUMERICAL MODEL}

In this work, the viability of the proposed theoretical model for signal degradation mainly caused by amplitude noise is evaluated with numerical simulation of single-channel RZ-ASK signal transmission at $40 \mathrm{Gbit} / \mathrm{s}$ bit rate in a typical dispersion-managed, terrestrial link [9], therefore operating a quasi-linear transmission regime. In the considered system, each amplifier span consists of $20 \mathrm{~km}$ effective core area enlarged positive dispersion fibre and negative dispersion fibre sections. The effective core area enlarged positive dispersion fibre (EE-PDF) has dispersion of $20 \mathrm{ps} /(\mathrm{nm} \mathrm{km})$, dispersion slope of $0.06 \mathrm{ps} /\left(\mathrm{nm}^{2} \mathrm{~km}\right)$ and effective core area of $110 \mu \mathrm{m}^{2}$. The negative dispersion fibre (NDF) has dispersion and dispersion slope of identical magnitude but with reversed sign, with effective core area of $30 \mu \mathrm{m}^{2}$. The fibres have attenuation of $0.2 \mathrm{~dB} / \mathrm{km}$ (EE-PDF) and $0.24 \mathrm{~dB} / \mathrm{km}$ (NDF). Each span also includes an erbium-doped fibre amplifier with a noise figure of $4.5 \mathrm{~dB}$ with the gain configured to compensate for the energy losses in the previous fibre section. Simulations are performed here for pseudo-random data sequences of $\left(2^{7}-1\right)$ length formed with Gaussian pulses. The standard pulse duty cycles of $33 \%, 50 \%$, and $67 \%$ are employed. A fifth-order Bessel filter with a cut-off frequency equal to the bit rate is used as a receiver low-pass filter. The results are averaged over a number of bit patterns to obtain accurate statistics of the in-line amplifier noise. The transmission performance of the system is degraded by both intrachannel nonlinear interactions-induced amplitude noise and timing jitter [9]. This presents a good model situation to evaluate the validity and illustrate the limits of the proposed methodology.

\section{RESULTS}

Shown in Fig. 2 is the evolution of the estimated ECP versus the transmission distance. The results of the approach commonly used for numerical simulations, where the EC is measured as the height of the rectangle of the largest area with a base of $0.2 T_{b}\left(T_{b}\right.$ is the bit period) that can be fitted within the open portion of the eye [2] (see Fig. 1), are also shown in Figs 2-4 .

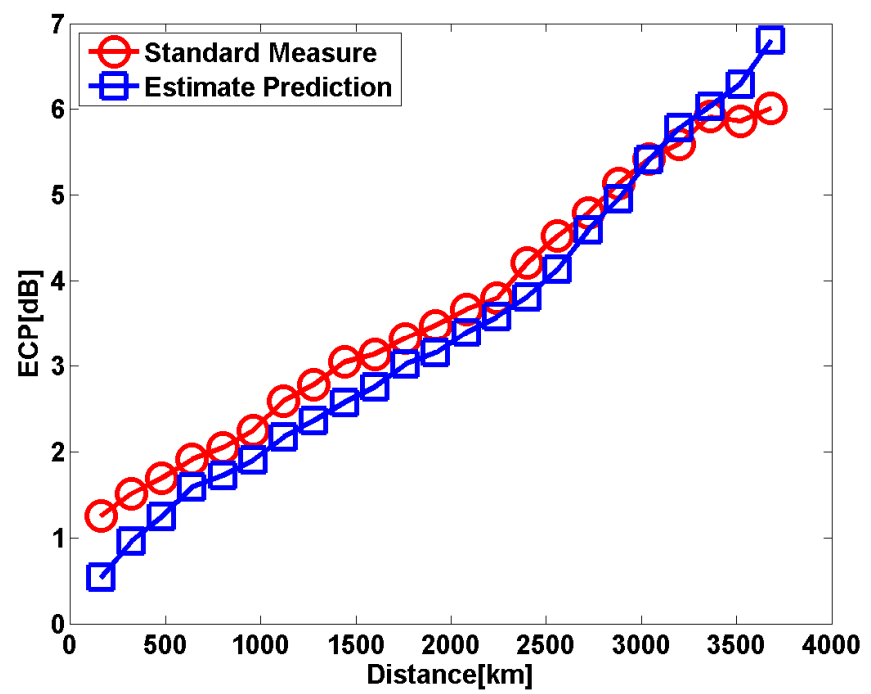

Figure 2: Eye-closure penalty versus distance for $40 \mathrm{Gbit} / \mathrm{s}$ RZ-ASK at $33 \%$ duty cycle 
We can see that there is good agreement between the model predictions of ECP and the results obtained from the standard numerical approach at the considered distances for all pulse duty cycles. Growing distance encourages the development of fibre nonlinearity, of which RZ-OOK pulses are particularly vulnerable [10], however we see here that our estimate is valid even at distances in excess of $3000 \mathrm{~km}$. The extension to long-haul transmission requires the consideration of the timing jitter, either using devices to offset the influence of timing jitter [9] or by including the statistical impact in the model. We do not consider either in this work.

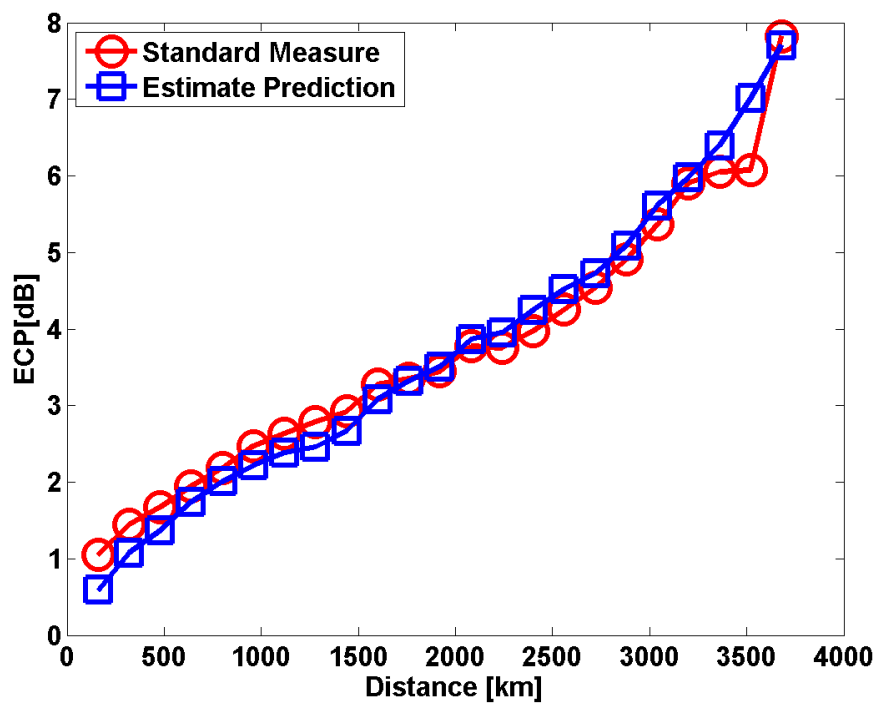

Figure 3: Eye-closure penalty versus distance for $40 \mathrm{Gbit} / \mathrm{s}$ RZ-ASK at $50 \%$ duty cycle

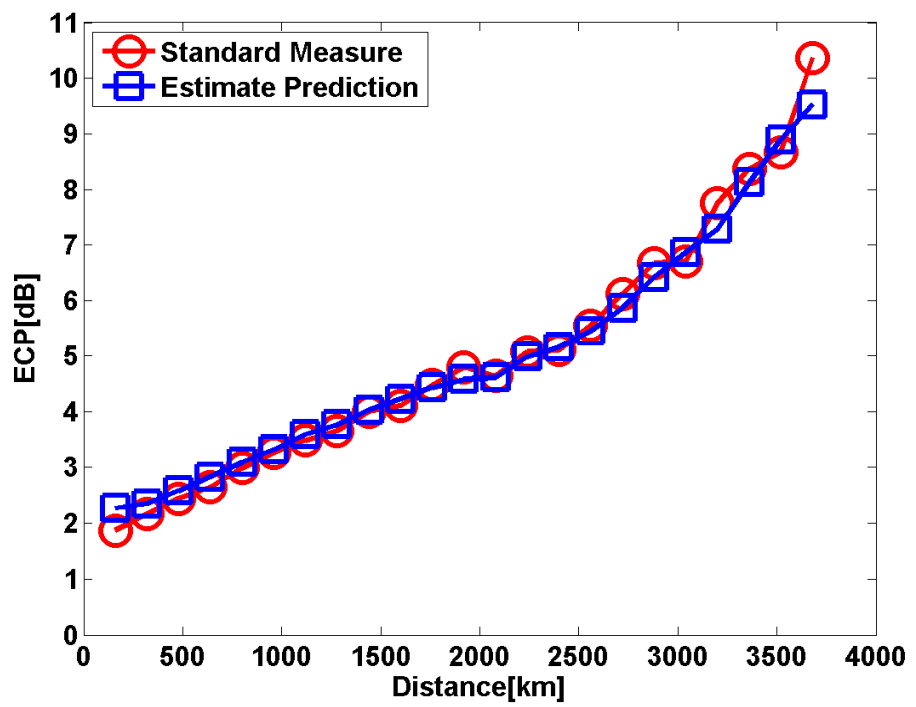

Figure 4: Eye-closure penalty versus distance for $40 \mathrm{Gbit} / \mathrm{s}$ RZ-ASK at $67 \%$ duty cycle

We observed in our study that, for the example system considered here, at distances larger than approximately $3500 \mathrm{~km}$ the ECP model yields incorrect predictions. The principle explanation for this is that the assumption of symmetry in the eye closing is less accurate when the effects of the accumulation of timing jitter and waveform distortion through the system become important. Evidently, the eye diagrams demonstrate the evolution of the eye closure across the 
transmission distance. This is the case across all duty cycles, as explained below in Fig. 5. We see that whilst the influence of timing jitter and amplitude fluctuations are comparable, the estimate works well. However, at increased distances timing jitter becomes the dominant source of distortion, causing the assumption of symmetry in the eye closure to break-down due to asymmetric eye closure

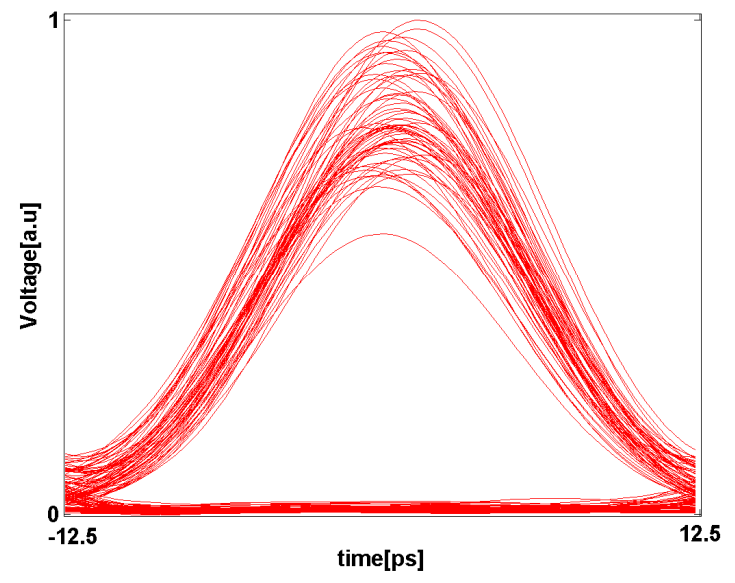

a)

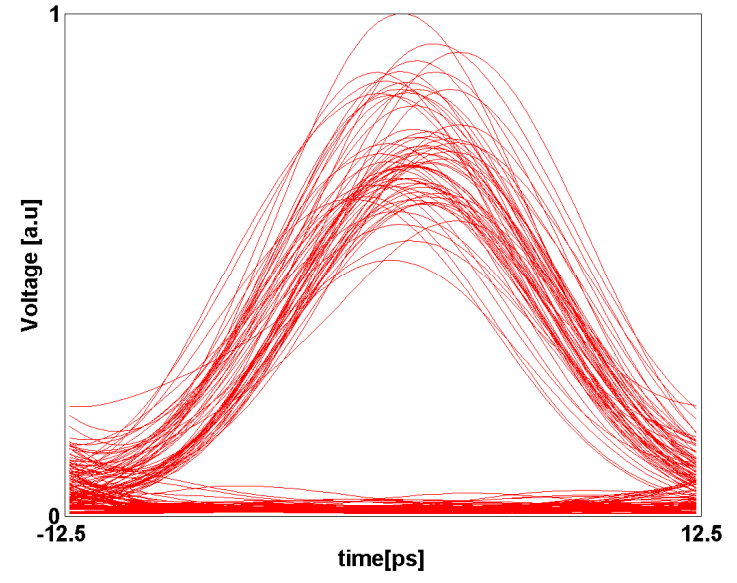

b)

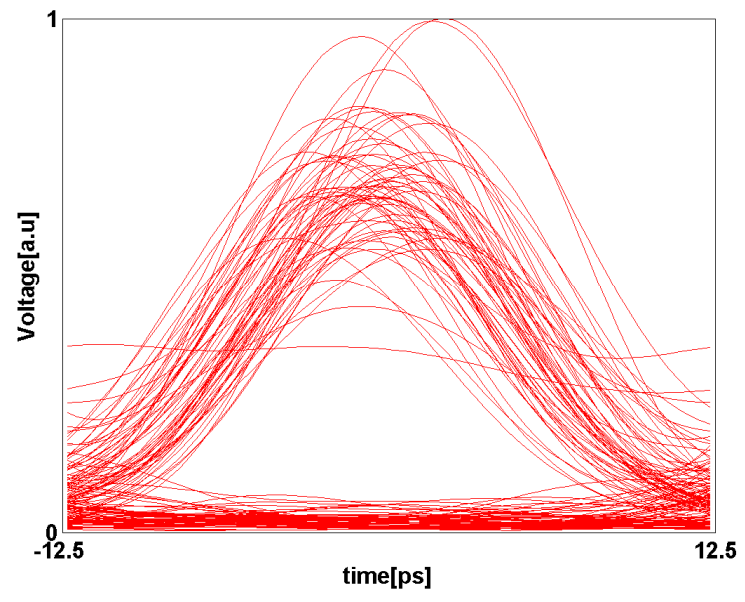

c)

Figure 5: 33\% Duty Cycle RZ-Pulses a) $1600 \mathrm{~km} \mathrm{b)} 2600 \mathrm{~km} \mathrm{c)3700} \mathrm{km}$

Studying the evolution of the eye diagrams corresponding to $33 \%$ duty cycle pulses, we see that the effects of IXPM and IFWM, namely amplitude noise and timing jitter, increase corresponding with distance. The evolution of eye closure penalty, given in Figs 2-4, demonstrates that for distances up to $3500 \mathrm{~km}$, the assumption of symmetric eye closure is upheld and our estimate is valid, as can be seen by Fig. 5 a) \& b). Distances beyond $3500 \mathrm{~km}$ present a rather different story, with timing jitter creating increasingly horizontal distortion on the transmitted pulses, as shown in Fig. $5 \mathrm{c}$ )

\subsection{Analysis of Probability Density Functions}

In order to extend our statistical analysis, we also wish to analyse the behaviour of the probability density function inside and outside of the validity of our estimate. We know that OOK, from an information theory point of view, can be modelled as a Binary Asymmetric Channel (BAC), so we cannot assume any symmetry across the ones and zero. 


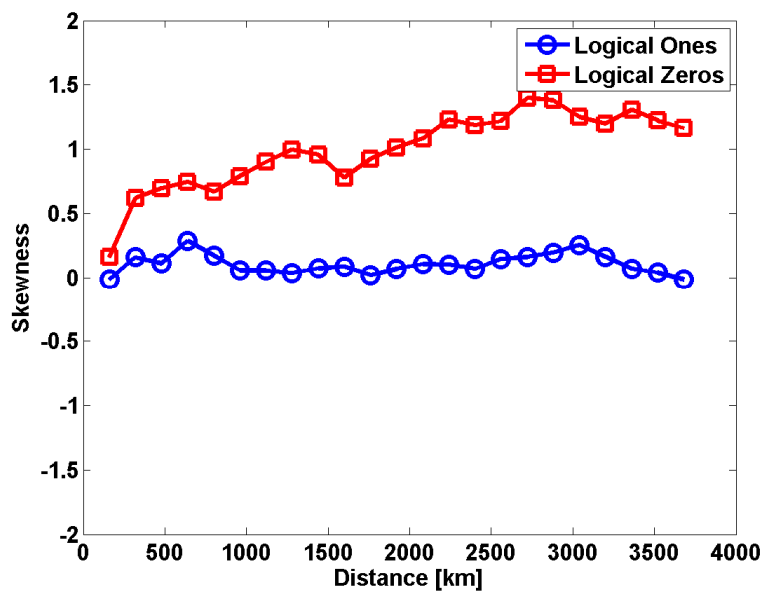

a)

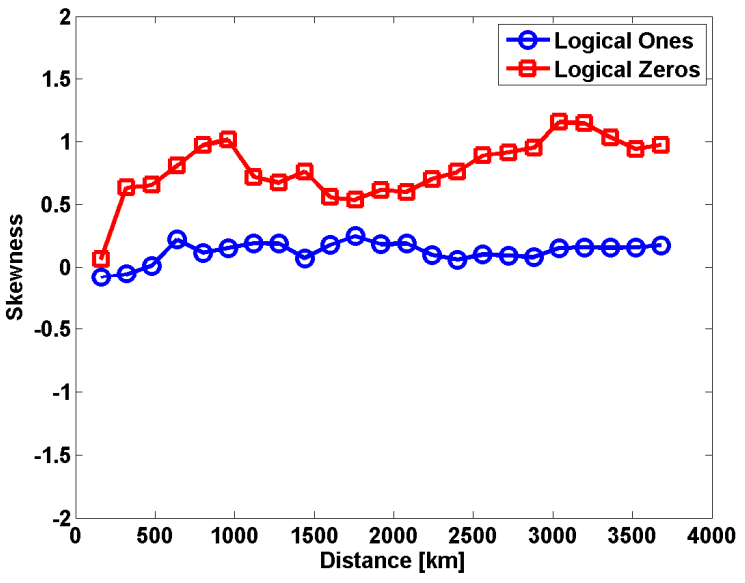

b)

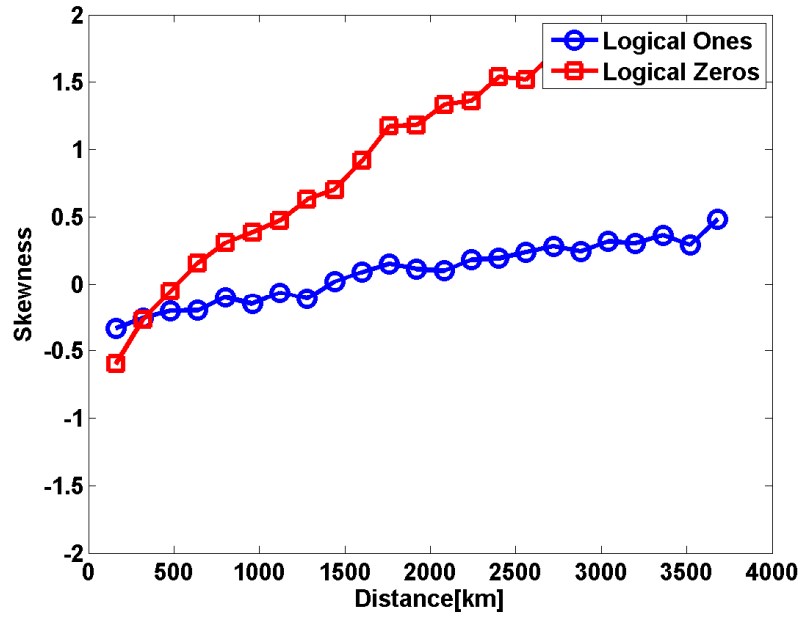

c)

Figure 6: Skewness of Voltage Distribution a) 33\% Duty Cycle b) 50\% Duty Cycle c) 67\% Duty Cycle

In-depth statistical analysis is supported via calculation of the skewness of the received voltage statistics. Skewness measures underlying asymmetry of the data and is calculated as the ratio between the third moment of the data and the cube of the standard deviation. Logically, if two distributions are symmetric to one another then the magnitude of their skewness must be identical. Such a feature doesn't concern the analysis of data generated from RZ-OOK channels because by its very nature we assume asymmetry between logical ones and logical zeros. However, the Gaussian distribution by its very nature is perfectly symmetric so such a measure will allow us to study the evolution of the shape of the data relative to the initial assumption.

Observing the skewness presented in Fig. 6, we see that symmetry within the logical ones remains largely constant throughout the transmission for all duty cycles considered. The distribution of logical zeros is, on the contrary, indicative 
of the accumulating noise within logical zeros when distance is increased. This is a result of four-wave mixing, which manifests as noise in the logical zeros and amplitude jitter in the logical ones. From this we can say that the growing influence of timing jitter causes asymmetry of the data and causes our assumption to become invalid.

Given our analysis of ECP is largely concerned with the extrema of the underlying voltage, it is also imperative to analyse the kurtosis of the voltage. Such a measure can also been seen as a measure of Gaussian behaviour. We see that when distance increases to over $3500 \mathrm{~km}$, the Gaussian structure of the data breaks down due to increased ISI and timing jitter dominating the transmission. We choose the distribution of the logical ones as a key indicator of the system behaviour and subsequently calculate the kurtosis (Fig. 7)

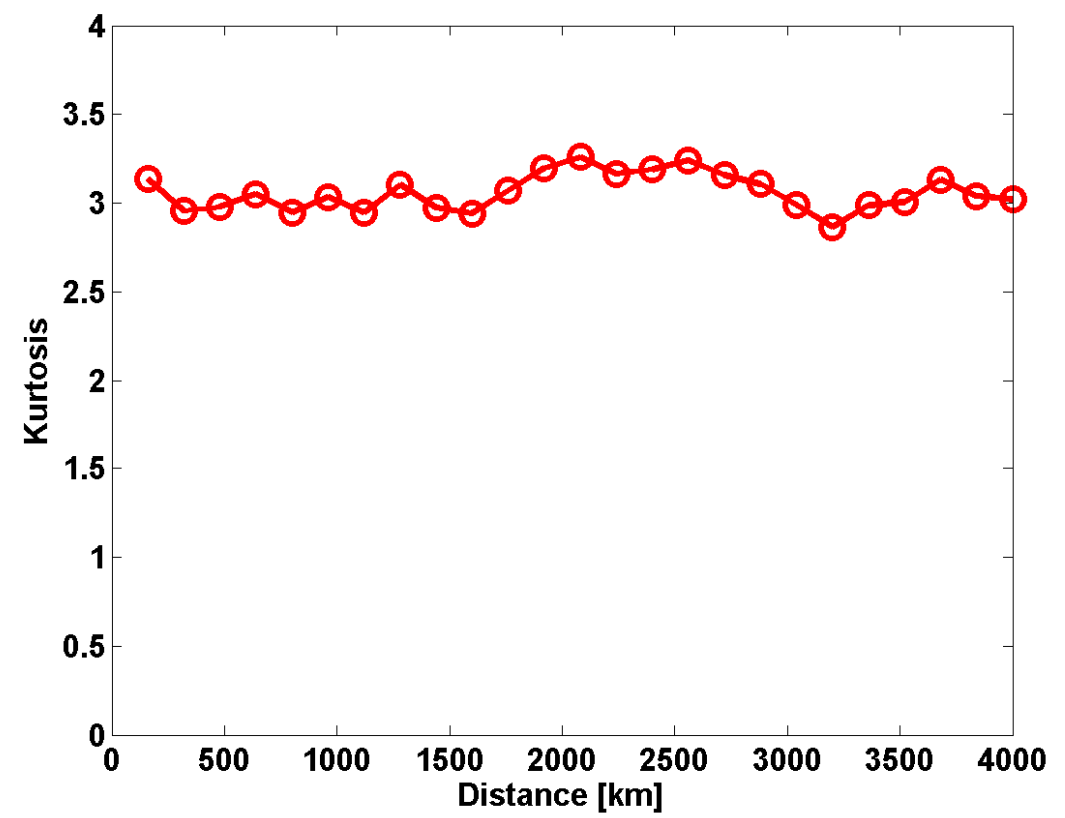

Figure 7: 50\% RZ Pulses Kurtosis of the Received Current Histograms at logical ones.

The kurtosis is calculated as the fourth moment divided by the variance squared, such a measure provides insight into the variation in the data and subsequent tail behaviour within potential distributive models. The kurtosis of Gaussian distributed data should be exactly 3. Data with kurtosis greater than 3 is known as lepto-kurtically distributed data and data with kurtosis less than 3 is known as platy-kurtically distributed data. Due to such a fact, we can say that from Fig. 7 that the distribution of received logical ones exhibits strong Gaussian behaviour throughout propagation. From this, previous examination of the skewness can reveal more about how the transmission behaves. Fig. 6 indicates strong skewing in the distribution of the logical zeros, yet almost no skewing in the logical ones. The results of both suggest that the logical ones remain as Gaussian distributed random variables but logical zeros have distance dependent statistical dynamics, with heavy asymmetry observed in Fig 6.

Examining the statistical structure of the logical zeros using the skewness can also reveal why the estimate failed at higher distances, asymmetry! We can say from Fig. 6 that the logical zeros are heavily skewed due to the noise and the growing influence of IFWM inducing energy changes in the logical zeros, which would otherwise be negligible in the logical ones.

\section{CONCLUSION.}

We have presented a simple theoretical method for a quick and easy estimation of the expected ECP in fibre-optic communication systems for signals mainly degraded by amplitude noise. Numerical simulation designed to test the model predictions have shown good agreement with the theoretical results over reasonable transmission distances, confirming the validity of the model over a certain penalty range. Further work is required to include "horizontal" effects 
in the modelling analysis. Despite such a limitation to our analysis, we show that asymmetry due to such effects is the source of failure in our analysis.

\section{REFERENCES}

[1] Essiambre, R.J. Mikkelsen and G.Raybon "Intra-channel Cross-Phase Modulation and Four-Wave Mixing in High-Speed TDM Systems" Electron. Lett 351576 (1999)

[2] Agrawal G.P., Lightwave Technology: Telecommunication Systems (John Wiley \& Sons, New Jersey, 2005).

[3] Downie, J.D "Relationship of Q-Penalty to Eye Closure Penalty for NRZ and RZ Signals with Signal Dependent Noise" J. Lightwave Tech. 23, 2031 (2005).

[4] Downie, J.D and Ruffin, A.B, "Analysis of Signal Distortion and Crosstalk Penalties Induced by Optical Filters in Optical Networks" J. Lightwave Tech. 21, 1876 (2003).

[5] Ho. K.-P, Chen. L.K. and Tong. F, "Modelling of Waveform Distortion due to Optical Filtering" IEEE J. Sel. Top. Quantum Electron. 6, 223 (2000).

[6] Abramovich, F. and Bayvel, P. "Some Statistical Remarks on the Derivation of BER in Amplified Optical Communication Systems" IEEE Trans. Communications. 451032 (1997)

[7] Marcuse, D. "Derivation of Analytical Expressions for the Bit-Error Probability in Lightwave Systems with Optical Amplifiers" J. Lightwave. Tech 81816 (1990)

[8] Bergano, N.S. Kerfoot, F.W and Davidson, C.R "Margin Measurements in Optical Amplifier Systems" IEEE Photon. Tech. Lett. 5, 304 (1993).

[9] Boscolo, S. Turitsyn, S.K. and Blow, K.J. "All-Optical Passive Quasi-Regeneration in Transoceanic 40 Gbit/s Return-to-Zero Transmission Systems with Strong Dispersion Management" Opt. Commun. 205, 30 (2002).

[10]Gnauck, A.H. and Winzer, P.J. "Optical Phase Shift Keyed Transmission" J.Lightwave Tech 23155 (2006) 